\title{
Vancomycin-resistant enterococci with reduced daptomycin susceptibility in Singapore: prevalence and associated factors
}

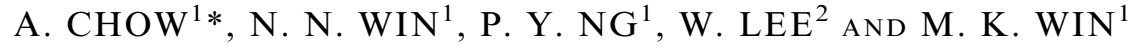 \\ ${ }^{1}$ Department of Clinical Epidemiology, Institute of Infectious Diseases and Epidemiology, Tan Tock Seng \\ Hospital, Singapore \\ ${ }^{2}$ Department of Laboratory Medicine, Tan Tock Seng Hospital, Singapore
}

Received 20 December 2015; Final revision 2 April 2016; Accepted 19 April 2016; first published online 13 May 2016

\section{SUMMARY}

Prevalence of vancomycin-resistant enterococci (VRE) and use of daptomycin are increasing in Asia. To determine the prevalence of daptomycin non-susceptible enterococci (DNSE) and understand factors associated with reduced daptomycin susceptibility in VRE, we conducted a case-control study in a 1600-bed adult tertiary hospital in Singapore. All VRE isolates from inpatients in 2012 were tested for daptomycin susceptibility. Patients with VRE isolates of daptomycin minimum inhibitory concentration (MIC) $\geqslant 3 \mu \mathrm{g} / \mathrm{ml}$ were classified as daptomycinreduced susceptible VRE (DRS-VRE) and those with daptomycin MIC $<3 \mu \mathrm{g} / \mathrm{ml}$ classified as daptomycin-susceptible VRE (DS-VRE). Medical records were reviewed for clinical and epidemiological data. None of $243 \mathrm{VRE}$ isolates had MIC $>4 \mu \mathrm{g} / \mathrm{ml}$ (DNSE). About half (135, $55 \%$ ) had reduced susceptibility to daptomycin (MIC 3-4 $\mu \mathrm{g} / \mathrm{ml}$ ). None in the DS-VRE group had prior exposure to daptomycin. After adjusting for age, gender, comorbidity, hospitalization duration, surgical history, indwelling device use, and duration of antibiotic exposure in the prior 3 months, $>1$ movement between wards [odds ratio (OR) $0 \cdot 35,95 \%$ confidence interval (CI) $0 \cdot 16-0 \cdot 74, P=0 \cdot 006]$ and minocycline resistance (OR 0.45, 95\% CI 0.25-0.84, $P=0 \cdot 011$ ) were independently associated with DRS-VRE. Our study suggests that daptomycin exposure, $>1$ movement between wards, and resistance to minocycline, were associated with reduced daptomycin susceptibility in VRE.

Key words: DNSE, epidemiology, reduced daptomycin susceptibility, risk factors, VRE.

\section{INTRODUCTION}

Vancomycin-resistant enterococci (VRE) have been recognized as a leading cause of hospital-acquired infections (HAI) globally since its emergence in 1986 in the UK and France [1]. VRE infections, with the organism's ability to acquire resistance and its intrinsic resistance to various antibiotics [2] coupled with

\footnotetext{
* Author for correspondence: A. Chow, 11 Jalan Tan Tock Seng, Singapore 308433.

(Email: Angela_Chow@ttsh.com.sg)
}

the scarcity of reliable antimicrobial therapy for the effective treatment of serious bacteremia [3], present a clinical dilemma for infectious disease physicians. Enterococci are the third most common organism causing nosocomial bloodstream infections, with vancomycin resistance observed in 60\% of Enterococcus faecium [4].

Daptomycin is one of a limited number of antibiotics with effective bactericidal activity against VRE [5]. Soon after its approval for use by the US Food and Drug Administration in 2003, reports of infections with daptomycin non-susceptible enterococci (DNSE) emerged and cases have been described in patients both with and 
without prior daptomycin exposure [6, 7]. The mechanism for the development of daptomycin non-susceptibility in enterococci has not been well elucidated [8], although there is the suggestion that DNSE colonization or infection increased with increasing daptomycin use $[9,10]$.

In Singapore, daptomycin was approved for use in 2008 and utilization has increased over the years [11]. Although daptomycin non-susceptible Staphylococcus aureus bacteraemia have been reported, little is known about the epidemiology of DNSE in Singapore and Asia. Since the first report of VRE in 1994, VRE rates have increased in Singapore, mirroring the pattern seen in Europe a decade earlier [12]. This study aims to determine the prevalence of DNSE in an Asian country with an increasing VRE prevalence and daptomycin use, and to understand the factors associated with reduced daptomycin susceptibility in VRE.

\section{METHODS}

We conducted a case-control study in a 1600-bed adult tertiary hospital in Singapore. The prevalence of clinical VRE infections in the hospital increased from 9.97/100 000 deaths and discharges in 2010 to $77 \cdot 11 / 100000$ in 2012. Correspondingly, daptomycin utilization increased by 6.4-fold from an average monthly defined daily dose $/ 1000$ patient-days of $0 \cdot 2$ in 2010 to 1.28 in 2012. The hospital had an active VRE screening programme where surveillance cultures were taken from 'high-risk' patients who were on renal dialysis or had a history of prior hospitalization in a foreign or private hospital in the preceding 12 months, on admission to the hospital. In addition, whenever a VRE infection was detected in an inpatient, contact screening of all patients who were in the same ward as the index patient for the duration of the patient's hospital stay prior to isolation was carried out.

Clinical and surveillance samples were plated on selective medium chromID VRE plates (bioMérieux, USA) and incubated aerobically at $35-37^{\circ} \mathrm{C}$ for $48 \mathrm{~h}$.

Suspect colonies were identified using matrixassisted laser desorption/ionization time-of-flight mass spectrometry (Bruker Daltonik GmHB, Germany); antimicrobial susceptibility testing was performed for ampicillin, ciprofloxacin, clindamycin, erythromycin, gentamicin (high level), linezolid, minocycline, penicillin, teicoplanin, and vancomycin, using the Vitek 2 System (bioMérieux) [13]. Select strains that were identified with low discrimination were confirmed using an in-house multiplex polymerase chain reaction based on primer sets published by Dutka-Malen et al. [14].
All VRE isolates from patients hospitalized from 1 January to 31 December 2012 were tested for daptomycin susceptibility using the Etest (bioMérieux). We defined daptomycin-reduced susceptible VRE (DRS-VRE), by using the criteria of the Clinical and Laboratory Standards Institute [15], as patients with a VRE isolate with daptomycin minimum inhibitory concentration (MIC) $\geqslant 3 \mu \mathrm{g} / \mathrm{ml}$ from screening or clinical cultures. Daptomycin-susceptible VRE (DS-VRE) was defined as patients with VRE isolates with daptomycin MIC $<3 \mu \mathrm{g} / \mathrm{ml}$. For patients with VRE isolated from multiple samples, we only included the first VRE isolate and examined exposures prior to the first isolation of VRE.

We reviewed medical records for clinical and epidemiological data, and recorded patients' demographics, comorbidities, prior healthcare exposures, current hospitalization experiences, antibiotic exposures and durations within the past 3 months, antimicrobial susceptibilities and genotypes of VRE. Additionally, Charlson's comorbidity index [16] was derived using coding algorithms. Clinical infection was determined as part of the hospital infection control unit's routine workflow, after review of patients for signs and symptoms of infection based on guidelines by the Centers for Disease Control and Prevention's National Healthcare Safety Network.

We computed odds ratios (ORs) and $95 \%$ confidence intervals (CIs), to compare differences in exposures and covariates between DRS-VRE and DS-VRE. Next, we constructed a multiple logistic regression model to control for confounding and assess for independent factors associated with DRS-VRE colonization or infection. We included variables decided a priori as factors associated with reduced daptomycin susceptibility based on prior knowledge from literature review to be associated with DNSE colonization and infection. We used the Hosmer-Lemeshow test to check for goodness of fit for the model. All statistical analyses were performed using Stata v. 12 (Stata Corp., USA).

\section{Ethical approval}

This study was approved by the Domain Specific Review Board of the National Healthcare Group, Singapore (DSRB- 2013/00228).

\section{RESULTS}

In total, 243 VRE isolates were analysed, including both VanA $(133,55 \%)$ and $\operatorname{VanB}(110,45 \%)$ genotypes. The majority of VREs $(213,88 \%)$ were isolated from screening cultures. Of the VRE clinical infections, urine was 


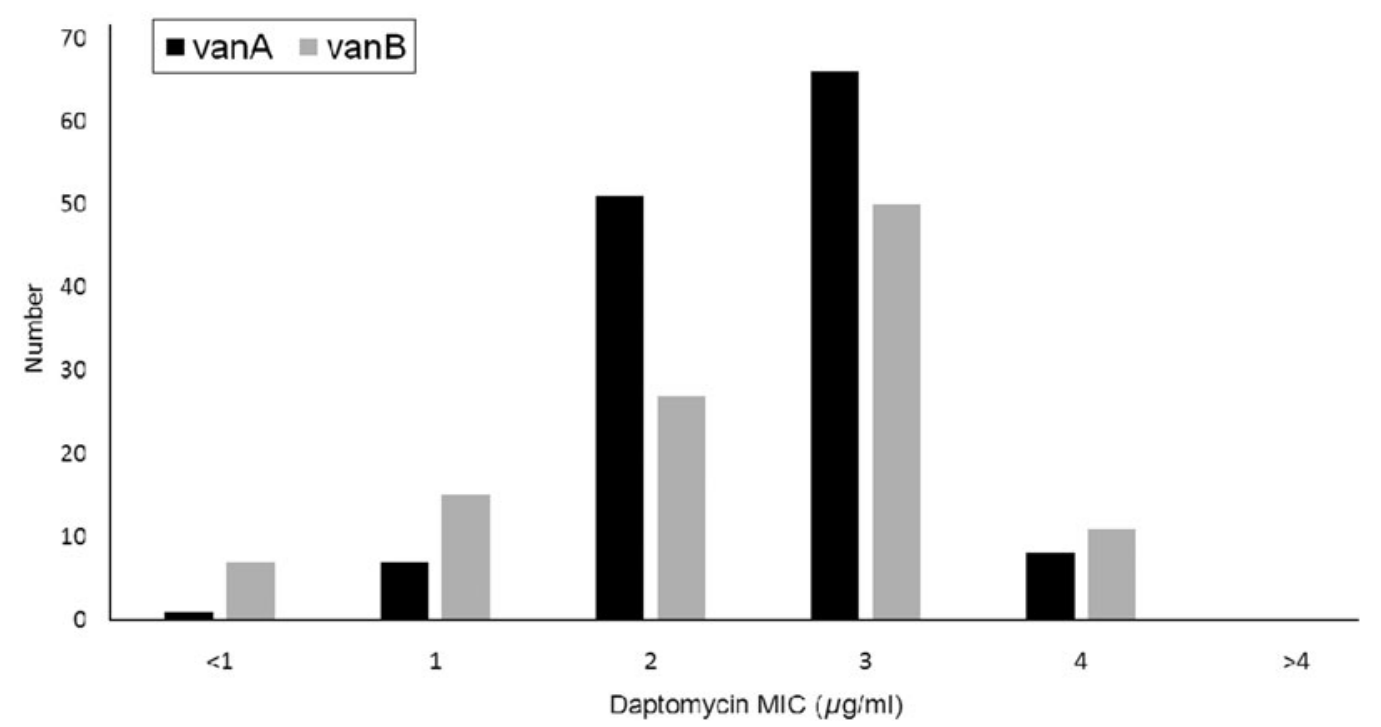

Fig. 1. Daptomycin minimum inhibitory concentration (MIC) levels by vancomycin-resistant enterococcus genotype.

found to be the main source $(67 \%)$. The daptomycin MICs were distributed similarly between genotypes (Fig. 1).

None of the isolates was determined to be daptomycin non-susceptible based on the Clinical Laboratory Standards Institute's antimicrobial susceptibility testing standards of MIC $>4 \mu \mathrm{g} / \mathrm{ml}$ [15]. However, about half of the patients $(135,55 \%)$ had reduced susceptibility to daptomycin with MIC $3-4 \mu \mathrm{g} / \mathrm{ml}$. Two-thirds (20/30) of clinical isolates had reduced daptomycin susceptibility compared to $54 \%(115 /$ 213) of screening isolates (OR $1 \cdot 70,95 \%$ CI $0 \cdot 72$ $4 \cdot 27, P=0 \cdot 191)$.

There was no difference in age and gender between the DRS-VRE and DS-VRE groups (Table 1). Both groups had similar prior healthcare exposures, with more than $70 \%$ having been hospitalized in the preceding year. Of the 135 DRS-VRE patients, more than two-thirds $(67 \cdot 4 \%)$ had a Charlson comorbidity score of $\geqslant 3$ and $76 \cdot 3 \%$ were immunosuppressed. The mean duration of hospital stay for all VRE patients was 26 days. Of note, DRS-VRE isolates $(14 \cdot 8 \%)$ were more likely to be from clinical infections than DS-VRE isolates $(9 \cdot 3 \%)$. Interestingly, patients who had $>1$ movement between wards during the hospitalization episode of VRE isolation (OR 0.57, 95\% CI $0.33-0.98, P=0.040$ ) were less likely to have DRS-VRE. Compared to DRS-VRE, DS-VRE patients were more likely to have experienced $>1$ movement between beds and to have been exposed to indwelling devices during hospitalization.
None of the DS-VRE patients in the study cohort had been exposed to daptomycin in the preceding 3 months, compared to three of the DRS-VRE patients. An antimicrobial exposure in the prior 3 months was not significantly different between the two groups, with exposure to penicillin being the longest. Duration of exposure to cephalosporin (OR 0.94, $95 \%$ CI $0 \cdot 89-1 \cdot 00, P=0 \cdot 044)$ was observed to be protective against DRS-VRE. Of note, VRE isolates that were resistant to minocycline were half as likely to have reduced daptomycin susceptibility as those who were not (OR $0 \cdot 49,95 \%$ CI $0 \cdot 28-0 \cdot 85, P=0 \cdot 012$ ). In both DRS-VRE and DS-VRE, $>90 \%$ of VRE isolates were resistant to erythromycin.

There was no difference in VRE genotypes, hospitalization exposure in the prior 1 year, number of movements between beds during hospitalization, number of types of antibiotics exposed to, exposure to surgical interventions, and admission to the intensive care unit, between DRS-VRE and DS-VRE patients. Four $(20 \%)$ out of the 20 DRS-VRE infected patients died during the hospitalization, compared to one $(10 \%)$ out of 10 DS-VRE patients [OR 2.00, 95\% CI $0 \cdot 26-15 \cdot 62, P=0 \cdot 640$ (Fisher's exact test)].

In multivariate analyses, the two independent factors associated with DRS-VRE isolation were $>1$ movement between wards (OR $0 \cdot 35,95 \%$ CI $0 \cdot 16-0 \cdot 74$, $P=0.006$ ) and resistance to minocycline (OR 0.45 , $95 \%$ CI $0 \cdot 25-0 \cdot 84, P=0 \cdot 011)$, after adjusting for age, gender, Charlson's comorbidity score $\geqslant 3$, duration of 
Table 1. Univariate and multivariate analyses of risk factors associated with daptomycin-reduced susceptible vancomycin-resistant enterococci (DRS-VRE)

\begin{tabular}{|c|c|c|c|c|c|c|}
\hline Factor & $\begin{array}{l}\text { DRS-VRE } \\
(N=135)\end{array}$ & $\begin{array}{l}\text { DS-VRE } \\
(N=108)\end{array}$ & OR $(95 \% \mathrm{CI})$ & $P$ & $\mathrm{aOR}(95 \% \mathrm{CI})$ & $P$ \\
\hline \multicolumn{7}{|l|}{ Demographics } \\
\hline Age, years, mean (S.D.) & $73 \cdot 0(14 \cdot 3)$ & $71 \cdot 5(14 \cdot 2)$ & $1 \cdot 01(0 \cdot 99-1 \cdot 03)$ & $0 \cdot 416$ & $1 \cdot 01(0 \cdot 99-1 \cdot 03)$ & $0 \cdot 447$ \\
\hline Female gender & $72(53 \cdot 3)$ & $50(46 \cdot 3)$ & $1 \cdot 33(0 \cdot 80-2 \cdot 20)$ & $0 \cdot 276$ & $1 \cdot 27(0 \cdot 70-2 \cdot 32)$ & $0 \cdot 418$ \\
\hline \multicolumn{7}{|l|}{ Comorbidities } \\
\hline Charlson score $(\geqslant 3)$ & $91(67 \cdot 4)$ & $63(58 \cdot 3)$ & $1 \cdot 48(0 \cdot 87-2 \cdot 50)$ & $0 \cdot 145$ & $1 \cdot 19(0 \cdot 66-2 \cdot 14)$ & $0 \cdot 552$ \\
\hline Immunosuppressive state* & $103(76 \cdot 3)$ & $74(68 \cdot 5)$ & $1 \cdot 48(0 \cdot 84-2 \cdot 61)$ & $0 \cdot 177$ & & \\
\hline Dementia & $26(19 \cdot 3)$ & $18(16 \cdot 7)$ & $1 \cdot 19(0 \cdot 62-1 \cdot 25)$ & 0.602 & & \\
\hline Liver disease & $9(6 \cdot 7)$ & $12(11 \cdot 1)$ & $0 \cdot 57(0 \cdot 23-1 \cdot 41)$ & $0 \cdot 225$ & & \\
\hline \multicolumn{7}{|l|}{ Exposure to healthcare settings and environments } \\
\hline Nursing-home resident & $18(17 \cdot 5)$ & $14(17 \cdot 1)$ & $1 \cdot 03(0 \cdot 48-2 \cdot 22)$ & $0 \cdot 943$ & & \\
\hline Hospitalization in past 1 year & $99(73 \cdot 3)$ & $78(72 \cdot 2)$ & $1 \cdot 06(0 \cdot 60-1 \cdot 87)$ & 0.847 & & \\
\hline \multicolumn{7}{|l|}{ Current hospitalization } \\
\hline Duration of stay, days, mean (s.D.) & $26 \cdot 2(42 \cdot 0)$ & $26 \cdot 4(27 \cdot 6)$ & $1 \cdot 00(0 \cdot 99-1 \cdot 01)$ & 0.975 & $1 \cdot 00(0 \cdot 99-1 \cdot 02)$ & $0 \cdot 471$ \\
\hline Movements between wards, mean (s.D.) & $1 \cdot 5(2 \cdot 4)$ & $1 \cdot 7(2 \cdot 5)$ & $0 \cdot 96(0 \cdot 86-1 \cdot 07)$ & $0 \cdot 442$ & & \\
\hline$>1$ movement between wards & $38(28 \cdot 1)$ & $44(40 \cdot 7)$ & $0.57(0 \cdot 33-0.98)$ & $0 \cdot 040$ & $0 \cdot 35(0 \cdot 16-0 \cdot 74)$ & 0.006 \\
\hline Movements between beds, mean (s.D.) & $2 \cdot 0(3 \cdot 1)$ & $2 \cdot 4(3 \cdot 1)$ & $0 \cdot 96(0 \cdot 88-1 \cdot 04)$ & $0 \cdot 277$ & & \\
\hline$>1$ movement between beds & $53(39 \cdot 3)$ & $53(49 \cdot 1)$ & $0 \cdot 67(0 \cdot 40-1 \cdot 12)$ & $0 \cdot 126$ & & \\
\hline Indwelling device use $\dagger$ & $10(7 \cdot 4)$ & $10(9 \cdot 3)$ & $0 \cdot 78(0 \cdot 31-1 \cdot 96)$ & $0 \cdot 602$ & $0 \cdot 74(0 \cdot 12-4 \cdot 58)$ & $0 \cdot 743$ \\
\hline Had surgery & $70(51 \cdot 9)$ & $51(47 \cdot 2)$ & $1 \cdot 20(0 \cdot 73-2 \cdot 00)$ & $0 \cdot 473$ & $1 \cdot 61(0 \cdot 88-2 \cdot 94)$ & $0 \cdot 131$ \\
\hline ICU admission & $16(11 \cdot 9)$ & $13(12 \cdot 0)$ & $0 \cdot 98(0 \cdot 45-2 \cdot 14)$ & $0 \cdot 965$ & $2 \cdot 21(0 \cdot 43-11 \cdot 29)$ & $0 \cdot 336$ \\
\hline \multicolumn{7}{|l|}{ Antibiotic exposure in past 3 months } \\
\hline Exposure to any antibiotics & $132(97 \cdot 8)$ & $102(94 \cdot 4)$ & $2 \cdot 59(0 \cdot 63-10 \cdot 60)$ & $0 \cdot 186$ & & \\
\hline Types of antibiotics exposed, mean (S.D.) & $3 \cdot 1(1 \cdot 5)$ & $3 \cdot 1(1 \cdot 8)$ & $1 \cdot 00(0 \cdot 88-1 \cdot 13)$ & 0.983 & & \\
\hline Aminoglycoside, days, mean (s.D.) & $2 \cdot 2(8 \cdot 5)$ & $1 \cdot 3(2 \cdot 5)$ & $1 \cdot 03(0 \cdot 97-1 \cdot 10)$ & $0 \cdot 350$ & $1 \cdot 06(0 \cdot 97-1 \cdot 16)$ & $0 \cdot 208$ \\
\hline Carbapenems, days, mean (s.D.) & $4 \cdot 2(8 \cdot 1)$ & $4 \cdot 0(7 \cdot 2)$ & $1 \cdot 00(0 \cdot 98-1 \cdot 04)$ & 0.863 & $1.02(0 \cdot 98-1 \cdot 06)$ & $0 \cdot 425$ \\
\hline Cephalosporin, days, mean (s.D.) & $1 \cdot 8(3 \cdot 5)$ & $3 \cdot 2(6 \cdot 6)$ & $0 \cdot 94(0 \cdot 89-1 \cdot 00)$ & $0 \cdot 044$ & $0 \cdot 95(0 \cdot 89-1 \cdot 02)$ & $0 \cdot 157$ \\
\hline Daptomycin, days, mean (S.D.) & $0 \cdot 1(0 \cdot 1)$ & $0 \cdot 0(0 \cdot 0)$ & $1 \cdot 79(0 \cdot 50-6 \cdot 35)$ & $0 \cdot 371$ & $1 \cdot 86(0 \cdot 46-7 \cdot 53)$ & $0 \cdot 382$ \\
\hline Fluroquinolone, days, mean (s.D.) & $2 \cdot 8(6 \cdot 5)$ & $4 \cdot 5(14 \cdot 1)$ & $0 \cdot 98(0 \cdot 96-1 \cdot 01)$ & $0 \cdot 234$ & $0 \cdot 98(0 \cdot 96-1 \cdot 01)$ & $0 \cdot 270$ \\
\hline Penicillin, days, mean (s.D.) & $11 \cdot 2(11 \cdot 8)$ & $9 \cdot 6(8 \cdot 6)$ & $1 \cdot 02(0 \cdot 99-1 \cdot 04)$ & $0 \cdot 221$ & $1 \cdot 02(0 \cdot 99-1 \cdot 05)$ & $0 \cdot 137$ \\
\hline Polymyxin B, days, mean (s.D.) & $0 \cdot 3(2 \cdot 9)$ & $0 \cdot 8(4 \cdot 5)$ & $0 \cdot 96(0 \cdot 88-1 \cdot 04)$ & $0 \cdot 290$ & $0 \cdot 95(0 \cdot 86-1 \cdot 04)$ & $0 \cdot 290$ \\
\hline Tetracycline, days, mean (S.D.) & $0 \cdot 1(0 \cdot 7)$ & $0 \cdot 1(0 \cdot 8)$ & $0 \cdot 97(0 \cdot 69-1 \cdot 37)$ & 0.875 & $0.92(0.59-1.43)$ & $0 \cdot 711$ \\
\hline Vancomycin, days, mean (s.D.) & $3 \cdot 7(7 \cdot 7)$ & $3 \cdot 8(6 \cdot 7)$ & $1 \cdot 00(0 \cdot 96-1 \cdot 03)$ & $0 \cdot 865$ & $0.98(0.94-1.04)$ & 0.575 \\
\hline \multicolumn{7}{|l|}{ Antibiotic resistance pattern of VRE } \\
\hline Resistant to teicoplanin & $73(54 \cdot 1)$ & $61(56 \cdot 5)$ & $0 \cdot 91(0 \cdot 55-1 \cdot 51)$ & $0 \cdot 708$ & & \\
\hline Resistant to minocycline & $31(23 \cdot 0)$ & $41(38 \cdot 0)$ & $0 \cdot 49(0 \cdot 28-0 \cdot 85)$ & $0 \cdot 012$ & $0 \cdot 45(0 \cdot 25-0 \cdot 84)$ & $0 \cdot 011$ \\
\hline Resistant to gentamicin & $63(47 \cdot 0)$ & $44(40 \cdot 7)$ & $1 \cdot 29(0 \cdot 77-2 \cdot 15)$ & $0 \cdot 329$ & & \\
\hline Resistant to erythromycin & $126(93 \cdot 3)$ & $106(98 \cdot 1)$ & $0 \cdot 26(0 \cdot 06-1 \cdot 25)$ & 0.093 & & \\
\hline \multicolumn{7}{|l|}{ Others } \\
\hline VanA VRE genotype & $74(54 \cdot 8)$ & $59(54 \cdot 6)$ & $0.99(0 \cdot 60-1 \cdot 65)$ & 0.977 & & \\
\hline Clinical infection & $20(14 \cdot 8)$ & $10(9 \cdot 3)$ & $1 \cdot 70(0 \cdot 76-3 \cdot 81)$ & $0 \cdot 195$ & & \\
\hline
\end{tabular}

OR, Odds ratio; aOR, adjusted odds ratio; CI, confidence interval.

Values are $n(\%)$ unless otherwise indicated.

The percentage is of patients for whom data were available, i.e. excluding the missing values.

* Includes one or more of the following: diabetes with and without chronic complication, leukaemia, lymphoma, any malignancy without metastases, metastatic solid tumour, connective tissue disease, HIV, renal disease.

$\dagger$ Central venous pressure line, endotracheal tube, peripherally inserted central catheter, urinary catheterization.

hospital stay, history of surgery, presence of indwelling devices, and duration of antibiotic exposure (to aminoglycoside, carbapenem, cephalosporin, daptomycin, fluoroquinolone, penicillin, polymyxin $\mathrm{B}$, tetracycline, vancomycin) in the prior 3 months.

\section{DISCUSSION}

Limited data is available on the risk factors associated with DNSE. To the best of our knowledge, this is the first study in Asia to assess the epidemiology of DNSE 
and factors associated with reduced daptomycin susceptibility in VRE. Daptomycin non-susceptibility (MIC >4) was not observed in our patient cohort, although $55 \%$ had reduced susceptibility to daptomycin (MIC 3-4 $\mu \mathrm{g} / \mathrm{ml}$ ). We observed similar daptomycin resistance patterns in genotypes VanA and VanB. A surveillance study on VRE in Taiwan during the same period also did not report any daptomycin nonsusceptibility [17]. The importance of reduced susceptibility to daptomycin has been under-recognized. The presence of mutations associated with daptomycin resistance have been recently observed in VRE isolates with daptomycin MIC 3-4 $\mu \mathrm{g} / \mathrm{ml}$, raising concerns about the effectiveness of daptomycin therapy in patients with such VRE infections [18]. Enterococcal isolates with daptomycin MIC 3-4 $4 \mathrm{~g} / \mathrm{ml}$ have been observed to harbour mutations in liaFSR, a threecomponent regulatory system controlling cell-envelope stress response, that have been linked with the emergence of daptomycin resistance in enterococci [19]. Another competing pathway associated with yycFGHIJ mutations have been reported to play an important role in the successful treatment of DRS-VRE infections and prevention of development into daptomycin non-susceptibility (MIC $>4 \mu \mathrm{g} / \mathrm{ml}$ ) [20].

In our study, we observed a relatively strong association between the duration of daptomycin exposure and daptomycin-reduced susceptibility (OR 1.86, 95\% CI 0.46-7.53), although statistical significance could not be reached due to the limited number of exposed patients. Another study conducted at the Detroit Medical Center similarly could not conclude that prior daptomycin exposure was a predictor for DNSE, due to the rare occurrence of prior daptomycin exposure [21]. Our findings support the observations of an earlier study at the University of Iowa Hospitals and Clinics which suggested the emergence of daptomycin non-susceptibility under the antimicrobial pressure of prior daptomycin exposure [9]. In that study, the number of patients with DNSE colonization or infection increased rapidly with the surge in daptomycin use.

Although immunosuppression and multiple comorbid conditions were reported to be associated with DNSE [9, 21], they were not observed to be associated with DRS-VRE in our study cohort. Our findings suggest that an immunosuppressive state might not be necessary for the development of daptomycin-reduced susceptibility. We also did not observe an increased risk of DRS-VRE in patients with indwelling devices, although exposure to such devices was previously reported to be an independent risk factor for DNSE [4].

Interestingly, we observed that VRE isolates that were resistant to minocycline were negatively associated with reduced daptomycin susceptibility. Our observation corroborates the finding of a recent study which reported minocycline resistance in VRE isolates that were susceptible to daptomycin from infected urinary stents in renal transplant patients [22]. Further studies are needed to better understand the mechanisms for antimicrobial resistance.

In our study, patients who had moved between wards were less likely to have DRS-VRE than those who had not (OR $0 \cdot 35,95 \%$ CI $0 \cdot 16-0 \cdot 74)$. This was not previously reported by studies on DNSE and more studies are warranted before any conclusions can be made.

Our study may have been limited by the small number of patients with prior daptomycin exposure. Nonetheless, we identified some interesting factors associated with reduced daptomycin susceptibility in VRE in an Asian country with increasing VRE prevalence and daptomycin use. Our findings suggest an association between daptomycin exposure and reduced daptomycin susceptibility.

\section{CONCLUSIONS}

DNSE was not observed in our study population; however, $55 \%$ had reduced daptomycin susceptibility with MIC 3-4 $\mu \mathrm{g} / \mathrm{ml}$. Such reductions in susceptibility to daptomycin have important clinical implications as mutations associated with daptomycin resistance have been observed. Our study suggests that daptomycin exposure, movement between wards, and resistance to minocycline were associated with reduced daptomycin susceptibility in VRE. Active surveillance for reduced susceptibility and non-susceptibility to daptomycin in VRE colonized or infected patients with prior daptomycin therapy is crucial, as the use of daptomycin increases against the backdrop of the VRE surge in Asia.

\section{ACKNOWLEDGEMENTS}

This study is supported by the Society of Infectious Diseases Singapore (SIDS)'s inaugural research fund.

\section{DECLARATION OF INTEREST}

None. 


\section{REFERENCES}

1. Uttley AH, et al. Vancomycin-resistant enterococci. Lancet 1988; 1: 57-58.

2. Murray BE. Vancomycin-resistant enterococcal infections. New England Journal of Medicine 2000; 342: $710-721$.

3. Landman D, Quale JM. Management of infections due to resistant enterococci: a review of therapeutic options. Journal of Antimicrobial Chemotherapy 1997; 40: 161170.

4. Wisplinghoff $\mathbf{H}$, et $\boldsymbol{a l}$. Nosocomial bloodstream infections in US hospitals: analysis of 24,179 cases from a prospective nationwide surveillance study. Clinical Infectious Diseases 2004; 39: 309-317.

5. Canton R, et al. A potential role for daptomycin in enterococcal infections: what is the evidence? Journal of Antimicrobial Chemotherapy 2010; 65: 1126-1136.

6. Fraher MH, et al. Daptomycin-resistant Enteroccoccus faecium in a patient with no prior exposure to daptomycin. Journal of Hospital Infection 2007; 65: 376-378.

7. Kelesidis T, et al. Case-control study comparing de novo and daptomycin-exposed daptomycin-nonsusceptible enterococcus infections. Antimicrobial Agents Chemotherapy 2012; 56: 2150-2152.

8. Palmer KL, et al. Genetic basis for daptomycin resistance in enterococci. Antimicrobial Agents Chemotherapy 2011; 55: $3345-3356$.

9. Storm JC, et al. Daptomycin exposure precedes infection and/or colonization with daptomycin nonsusceptible enterococcus. Antimicrobial Resistance and Infection Control 2012; 1: 19.

10. Kelesidis T, et al. Daptomycin nonsusceptible enterococci: an emerging challenge for clinicians. Clinical Infectious Diseases 2011; 52: 228-234.

11. Hsu LY, et al. Six cases of daptomycin-non-susceptible Staphylococcus aureus bacteraemia in Singapore. Journal of Medical Microbiology 2010; 59: 1509-1513.

12. Molton JS, et al. The global spread of healthcareassociated multidrug-resistant bacteria: a perspective from Asia. Clinical Infectious Diseases 2013; 56: 13101318.

13. Abele-Horn M, et al. Validation of VITEK 2 version 4.01 software for detection, identification, and classi- fication of glycopeptide-resistant enterococci. Journal of Clinical Microbiology 2006; 44: 71-76.

14. Dutka-Malen S, Evers S, Courvalin P. Detection of glycopeptide resistance genotypes and identification to the species level of clinically relevant enterococci by PCR. Journal of Clinical Microbiology 1995; 33: 1434.

15. Clinical and Laboratory Standards Institute. Performance standards for antimicrobial susceptibility testing: 20th informational supplement, 2012; Wayne, PA (CLSI document M100-S22).

16. Charlson ME, et al. A new method of classifying prognostic comorbidity in longitudinal studies: Development and validation. Journal of Chronic Diseases 1987; 40: 373-383.

17. Tse H, et al. National surveillance on vancomycinresistant Enterococcus faecium in Taiwan: emergence and widespread of ST414 and a Tn1546-Like element with simultaneous insertion of IS1251-like and IS1678. PLOS ONE 2014; 9: e115555.

18. McKinnell JA and Arias CA. Linezolid vs daptomycin for vancomycin-resistant enterococci: the evidence gap between trials and clinical experience [Editorial Commentary]. Clinical Infectious Diseases 2015; 61: 879-882.

19. Munita JM, et al. Correlation between mutations in liaFSR of Enterococcus faecium and MIC of daptomycin: revisiting daptomycin breakpoints. Antimicrobial Agents and Chemotherapy 2012; 56: 4354-4359.

20. Humphries RM, et al. Genotypic and phenotypic evaluation of the evolution of high-level daptomycin nonsusceptibility in vancomycin-resistant Enterococcus faecium. Antimicrobial Agents and Chemotherapy 2012; 56: 6051-6053.

21. Judge T, et al. Epidemiology of Vancomycin-resistant enterococci with reduced susceptibility to daptomycin. Infection Control and Hospital Epidemiology 2012; 33: $1250-1254$.

22. Descourouez JL, et al. Fosfomycin synergy in vitro with amoxicillin, daptomycin, and linezolid against vancomycin-resistant enterococcus faecium from renal transplant patients with infected urinary stents. Antimicrobial Agents and Chemotherapy 2012; 57: $1518-1520$. 